How to reference this article

Ślarzyńska, M. (2020). Cristina Campo e la ricezione di Gustaw Herling-Grudziński in Italia. Italica Wratislaviensia, 11(1), 215-233.

DOI: http://dx.doi.org/10.15804/IW.2020.11.1.09

Małgorzata Ślarzyńska

Uniwersytet Kardynała Stefana Wyszyńskiego w Warszawie

m.slarzynska@uksw.edu.pl

ORCID ID: 0000-0001-5948-698X

\title{
CRISTINA CAMPO E LA RICEZIONE DI GUSTAW HERLING-GRUDZIŃSKI IN ITALIA
}

\author{
CRISTINA CAMPO AND THE RECEPTION \\ OF GUSTAW HERLING-GRUDZIŃSKI IN ITALY
}

\begin{abstract}
Among other records, the Archive of Gustaw Herling-Grudziński in Naples holds Cristina Campo's unpublished letters to him. The letters bear witness to a literary relation between the two writers and intellectuals which began when Pale d'altare, a volume of short stories by HerlingGrudziński, was translated into Italian and published in Italy in 1960. Cristina Campo, a poet, essayist, translator and prominent figure in $20^{\text {th }}$-century Italian literature, was enthusiastic about HerlingGrudziński's volume. She comprehensively discussed it in an insightful review entitled 'La torre e l'isola,' published in Il Punto in 1961. Her review marked Herling-Grudziński's debut in Italy and became a highly influential factor in the Italian reception of his literary works. The author himself regarded it as one of the most important critical texts addressing his writings. The aim of my paper is to highlight the relevance of Cristina Campo to the Italian reception of Herling-Grudziński's works and to illumine the relations between the Polish writer and his Italian critic through the unpublished letters preserved in the Herling-Grudziński Archive. The paper also refers to other reviews of Pale d'altare, which were offered by Elèmire Zolla, Roberto Calasso, Margherita Guidacci and Leone Traverso, associated with the same literary circle, and shows the impact of Campo's enthusiasm on some of these appraisals.
\end{abstract}

Keywords: Cristina Campo, Gustaw Herling-Grudziński, Pale d'altare, correspondence, reception 


\section{Wel Diario scritto di notte, in data 20 marzo 1999, Gustaw Herling- 1 Grudziński dedica un intenso ricordo a Cristina Campo, nominan- dola al primo posto tra i "buoni critici":}

Avere buoni critici: il sogno di ogni scrittore. Nel mio caso si è avverato. Non li elencherò tutti, elencherò solo quelli che mi sono più vicini, che sono diventati anche miei amici.

Per prima nominerò Cristina Campo, proveniente da Firenze, all'anagrafe Vittoria Guerrini. L'ho conosciuta a Roma, poco dopo essermi stabilito in Italia. Era una donna adorabile e bella, di inconsueta sensibilità, incurabilmente malata di cuore dai primi anni di giovinezza. Era così malata che seguendo i consigli dei medici smise di alzarsi dal letto per evitare che quel suo cuore malato e fragile si frantumasse in movimento. Continuò a lavorare a letto fino alla morte precoce. Faceva traduzioni eccellenti (come Venezia salva e gli studi sui temi greci di Simone Weil, e le poesie di Emily Dickinson) $)^{1}$, scriveva saggi molto acuti che oggi entrano nel canone della saggistica italiana. Amava la poesia, la musica (specialmente quella gregoriana) e la pittura. Talvolta scriveva poesie ed emulazioni delle classiche fiabe orientali. Dopo che furono pubblicate le mie Pale d'altare ne scrisse in un noto settimanale romano; il suo saggio è stato poi usato come prefazione della seconda edizione di questi racconti, con i disegni di Lebenstein (allora era già morta). Debuttai in Italia sotto una stella felice e triste (HerlingGrudziński, 2012, p. 922)².

L'articolo di Cristina Campo, a cui Herling accenna, fu pubblicato nella rivista romana "Il punto" 1'11 marzo 1961. Si trattava di una re-

1 Gustaw Herling si riferisce rispettivamente a Venezia salva di Simone Weil tradotta da Cristina Campo (Weil, 1963), alla sua traduzione del saggio di Weil, L'Iliade o il poema della forza (Weil, 1974 [1967]) e alle traduzioni di Emily Dickinson originariamente pubblicate ancora sotto il nome di Vittoria Guerrini nel "Corriere dell'Adda" (Dickinson, 1953) e poi raccolte insieme alle altre inedite traduzioni di Dickinson nel volume La Tigre Assenza (Campo, 1991).

2 Trad. del frammento in italiano mia - MŚ. I frammenti del terzo volume del Diario scritto di notte tradotti da Vera Verdiani e pubblicati per la curatela di Krystyna Jaworska nel Meridiano di Herling nel 2019, non comprendono il frammento citato sopra. Nessun cenno al testo e al ruolo di Cristina Campo si trova nell'importante studio sulle relazioni di Herling con la letteratura italiana recentemente pubblicato da M. Śniedziewska (Śniedziewska, 2019). 
censione al volume di Herling Pale d'altare ${ }^{3}$, uscito l'anno prima per l'editore Silva (Herling, 1960), che raccoglieva i racconti La torre e L' $i$ sola. La "piccola nota", come la chiama la Campo in una delle lettere a Herling del carteggio inedito di Cristina Campo conservato nell'Archivio di Gustaw Herling, sarebbe stata poi ristampata come prefazione della seconda edizione italiana del racconto La torre in volume, insieme al racconto $\mathrm{Il} \mathrm{miracolo,} \mathrm{nel} 1990$ (Campo, 1990) (questa stessa edizione, compreso lo scritto introduttivo di Cristina Campo, sarebbe stata tradotta anche in francese e pubblicata due anni più tardi [Campo, 1992]). È importante anche notare che è proprio il testo della Campo a inaugurare la fortuna letteraria di Gustaw Herling in Italia: come sottolinea l'autore stesso nella nota di diario, egli debuttò in Italia "sotto una stella felice e triste", cioè la stella di Cristina Campo

$\mathrm{Nel}$ contesto della ricezione di Herling in Italia Cristina Campo rappresenta un riferimento costante. Lo testimonia, fra l'altro, l'edizione italiana di alcuni frammenti del Diario scritto di notte (1992), dove una frase dell'articolo di Cristina Campo viene citata nel risvolto di copertina: "Come i narratori del XVIII secolo, Herling sa ancora tessere, in una sola, inconsutile trama, paesaggio sentimento sogno e moralità; e le grandi parole cerimoniali dell'orrore e della pietà traversano il suo discorso con la stessa naturalezza del vento autunnale fra gli alberi e della pioggia sui vetri"'. Un esempio ancora più attuale della grande risonanza del breve scritto di Cristina Campo si può trovare anche nell'articolo di Roberto Saviano su Gustaw Herling, in cui l'autore di Gomorra riporta lo stesso frammento (Saviano, 2009, p. 230) $)^{5}$. Il medesimo bra-

3 La versione originale polacca, Skrzydła oltarza, contenente i racconti La torre e Pietà dell'isola, è stata pubblicata a Parigi dall'Instytut Literacki. Il racconto La torre è stato prima pubblicato sulla rivista parigina "Kultura" (1958, n. 12). La pubblicazione del volume Pale d'altare in italiano suscitò interesse da parte di vari letterati italiani, tra cui, oltre alla stessa Cristina Campo, anche Ignazio Silone, Nicola Chiaromonte, Ennio Flaiano. Vedi M. Herling, 2019, pp. CXLII-CXLIII.

4 C. Campo, La torre e l'isola, frammento citato nella nota sulla copertina di Herling-Grudziński (1992).

5 Il testo di Saviano, che riguardava soprattutto la nuova edizione di Un mondo a parte, è stato originariamente pubblicato su "Pulp", n. 48, marzo/aprile 2004, 
no venne infine citato nella già menzionata nota del Diario di Herling, il quale conclude proprio con questa citazione la sua riflessione sui suoi "buoni critici"'.

Nell'ambito della ricezione italiana di Herling, così come nello sguardo dell'autore stesso sulla ricezione delle sue opere, il breve saggio di Campo risulta di fondamentale importanza. In Polonia, al contrario, il contributo di Cristina Campo alla fortuna di Herling è stato finora quasi del tutto trascurato, forse anche perché le opere della scrittrice non sono mai state tradotte in polacco, né si menzionano le sue opere nei manuali polacchi dedicati alla storia della letteratura italiana. Lo scopo di questo articolo e rendere conto del ruolo e dell'importanza di Cristina Campo nella ricezione dell'opera di Gustaw Herling-Grudziński in Italia, nonché presentare il rapporto che è nato tra lo scrittore polacco e la sua critica italiana attraverso le lettere inedite della Campo a HerlingGrudziński.

Il saggio di Cristina Campo, poi confluito nel volume pubblicato postumo Sotto falso nome (Campo, 1998), mostra non solo una lettura straordinariamente acuta dei due racconti di Herling, ma anche quello stile inconfondibile, di rara perfezione, che le ha fatto guadagnare un posto sicuro tra le maggiori autrici del Novecento italiano ${ }^{7}$. La perfezione,

e in seguito nel blog "Nazione Indiana", 3 giugno 2004 (https://www.nazioneindiana. com/2004/06/03/su-gustaw-herling/).

${ }^{6}$ Nella traduzione polacca dello stesso Herling: "Jak narratorzy z XVIII wieku Herling potrafi jeszcze połączyć w jednej fabule krajobraz, uczucie, sen i morał; i wielkie ceremonialne słowa zgrozy i litości znaczą jego opowiadania z taką samą naturalnością jak jesienny wiatr między drzewami czy deszcz na szybach okien" (Herling-Grudziński, 2012, p. 923).

${ }^{7}$ In Italia l'attenzione nei riguardi di Cristina Campo e della sua opera è iniziata a crescere soprattutto grazie all'uscita nel prestigioso catalogo della "Biblioteca" Adelphi del volume postumo Gli imperdonabili (Campo, 1987), seguito poi da La Tigre Assenza (Campo, 1991) e da Sotto falso nome (Campo, 1998). Per un primo approfondimento su Cristina Campo, si può vedere l'unica (finora) biografia Belinda e il mostro. Vita segreta di Cristina Campo firmata dalla giornalista Cristina De Stefano (De Stefano, 2002) e il sito Internet gestito dal professor Alberto Donati (www. cristinacampo.it), in cui si possono trovare molti materiali utili riguardanti la scrittrice. Tra i volumi monografici su Cristina Campo, si veda almeno quello di Monica Farnetti Cristina Campo (Farnetti, 1996). Cristina Campo è considerata anche una 
intesa in chiave estetica ed etica, è stata la qualità letteraria a cui Cristina Campo aspirò di più. Esitava sempre prima di pubblicare qualunque cosa e per pubblicare doveva avere la sensazione che il suo testo fosse perfetto. Rimane famosa la sua frase pubblicata nel risvolto di copertina del suo volume di saggi Il flauto e il tappeto (Campo, 1971), nella quale disse di sé stessa: "Ha scritto poco e le piacerebbe avere scritto meno".

La celebre frase può essere applicata anche al suo testo dedicato a Herling, in cui in breve spazio riesce a fornire un contributo sostanzioso, pieno di intuizioni brillanti, e forse uno dei più importanti nell'ambito della critica europea su Herling. Non trovando quasi nessuno scrittore affine ("la stirpe di Herling è tutt'altra") tra gli altri autori pubblicati e pubblicizzati dall'editore Silva sul foglietto inserito nel suo volume (Conrad, Saint-Exupéry, Graham Greene, Camus e Bernanos, l'unico che potrebbe essere considerato congeniale a Herling, "ma per ragioni del tutto particolari"), Campo popone l'accostamento con i moralisti del XVIII e XIX secolo. L'autrice menziona, in particolare, Xavier De Maistre (Herling si era esplicitamente ispirato a La lépreux de la cité d'Aoste), e chiama in causa anche De Quincey e Maurice de Guérin.

Un peso particolare ha il riferimento a Borges: "Fatti questi nomi, non so se non darei a Herling, nella mia antologia, il posto accanto a quello di un narratore che intimamente è tanto diverso da lui quanto può esserlo soltanto il fratello della fiaba: Jorge Luis Borges" (Campo, 1998, p. 76). Cristina Campo suggerisce un'analogia tra "il gelido pitagorismo" di Borges e "la tenebrosa cattolicità" di Herling. Tutti e due non riescono, secondo lei, a strutturare un racconto in modo che non sia estremamente simmetrico, "dove nulla resterà senza una risposta, senza un'eco fatale e rivelatrice; dove il mistero si manifesterà, come deve, nel silenzio degli specchi e nei cicli della spirale" (Campo, 1998, p. 77). L'accostamento di Herling a Borges è senz'altro una delle intuizioni

grande scrittrice di lettere: cfr. Lettere a Mita (Campo, 1999), Lettere a un amico lontano (Campo, 1998), Caro Bul. Lettere a Leone Traverso (1953-1967) (Campo, 2007), Il mio pensiero non vi lascia. Lettere a Gianfranco Draghi e ad altri amici del periodo fiorentino (Campo, 2011), Carteggio di Cristina Campo e Alessandro Spina (Campo \& Spina, 2007), Se tu fossi qui. Lettere a Maria Zambrano 1961-1975 (Campo, 2009), Un ramo già fiorito. Lettere a Remo Fasani (Campo, 2010). 
forse più penetranti dell'articolo. Herling comunque, secondo la scrittrice, avrebbe un vantaggio su Borges: è capace di usare "le grandi parole cerimoniali dell'orrore e dalla pietà" con una piena naturalezza, come, appunto, solo i narratori del Settecento erano in grado di fare.

Campo riassume brevemente la trama de La Torre e accenna alla storia raccontata nella Pietà dell'isola, soprattutto per mostrare le simmetrie e i riferimenti interni nelle trame dei racconti:

in realtà non sono più gli eventi, non sono neppure i luoghi (l'isola nell'isola come la torre nella torre: la Certosa nell'isola e il sordomuto nella Certosa) a rispondersi tragicamente, ma piuttosto gli inesprimibili, spesso impensabili pensieri degli uomini: peccato contro peccato, malattia contro malattia, afflizione contro afflizione (Campo, 1998, p. 80).

A detta di Cristina Campo, nei suoi racconti, Herling unisce in modo naturale concetti e elementi opposti, come il peccato e la gloria, la lebbra e il cielo, articolando la sua "millenaria liturgia funebre". Un altro scrittore, oltre a Borges, che Campo collega a Herling nel suo personale canone letterario, è Furio Monicelli, l'autore del Gesuita perfetto (Campo, 1998, p. 80). La scrittrice termina la sua lettura dei racconti di Herling con una frase di Kafka, citata nella Pietà dell'isola: "Siamo come bambini smarriti nella selva. Quando tu stai dinanzi a me e mi guardi, che cosa sai dei miei dolori e che cosa so io dei tuoi? E se io cadessi dinanzi a te e piangessi e ti raccontassi, ne sapresti di me più che dell'inferno, quando ti dicono che brucia e mette i brividi" (Campo, 1998, p. 81), e loda le capacità di Herling di riconoscere una poesia in questa frase proveniente dall'epistolario di Kafka.

Cristina Campo era entrata in contatto con Gustaw Herling tramite "Tempo Presente", a cui entrambi collaboravano, ma anche per il tramite di Elena $\mathrm{Croce}^{8}$. La ristretta cerchia degli amici che frequentavano Cristina Campo e l'allora suo compagno Elémire Zolla, si manteneva a una certa distanza dal centro della vita letteraria, in una posizione "vo-

8 Vedi Note in: Campo, 2007, p. 191. Cristina Campo associa Herling a Elena Croce anche in una delle lettere a Leone Traverso, vedi Lettera di Cristina Campo a Leone Traverso [n. 101], del 11 maggio 1961, in: Campo, 2007, p. 112. 
lutamente isolat[a], un po' elitari[a], per definizione estrane[a] a ogni tema che sia anche lontanamente alla moda" (De Stefano, 2002, p. 111). Tra gli amici comuni di Cristina Campo e Zolla - Elena Croce, Giovanni Macchia, Mario Bortolotto, Marìa Zambrano, il giovane Roberto Calasso, Livio Bacchi, Juan Rodolfo Wilcock e Pietro Citati - vi era anche Gustaw Herling9.

Nella lettera a Gustaw Herling citata all'inizio, la prima tra quelle conservate nell'Archivio di Herling, Cristina Campo informa lo scrittore della spedizione del numero del "Punto" con la sua recensione a Pale d'altare. Pur essendo stata registrata come prima lettera del carteggio dagli $\operatorname{archivisti~}^{10}$ (reca la numerazione 1), in base alla sua datazione (12 febbraio1961) risulta in realtà essere la seconda in ordine cronologico. La data 12 febbraio 1961 messa per iscritto in testa della lettera da Cristina Campo è sbagliata e dovrebbe essere 12 marzo 1961, perché: a) il timbro postale sulla busta, conservata insieme alla lettera nell'Archivio (e descritta con il numero 1a), riporta la datazione 15 marzo 1961 ; b) la lettera riguarda il già più volte menzionato articolo della Campo pubblicato su "Il Punto", che uscì l'11 marzo 1961. Cristina Campo, dunque, scrisse la lettera a Herling il giorno dopo la pubblicazione dell'articolo (non avrebbe certo potuto spedirla il mese precedente, quando l'articolo non era ancora uscito).

La lettera inizia con la formula "Caro Amico", mentre in tutte le altre si rivolge a lui, chiamandolo "Caro Herling". La lettera è piena di ammirazione verso il volume di racconti di Herling:

Speravo di poterLa ringraziare molto prima, per questa opera che mi ha tanto colpita, e di poterlo fare molto meglio. Purtroppo il giornale ha tardato a pubblicare la nota e il poco spazio mi ha costretta ad uno spessore molto

9 Elencato nel gruppo romano anche in: Pertignani, 2012. L'amicizia tra Cristina Campo, Elémire Zolla e Gustaw Herling viene confermata nella testimonianza di Piero Craveri: "Anche a Roma il sodalizio era ristretto: Nicola Chiaromonte, Ignazio Silone, e poco altro. Ricordo che Élemire Zolla e Cristina Campo erano suoi amici” (Craveri, 2015, p. 199).

10 Il frammento della lettera di Campo pubblicato nel «Meridiano» di Gustaw Herling risulta citato con la data 12 febbraio (II) 1961, interpretata come 12 novembre 1961. Vedi Herling, 2019, p. CXLIII. 
sottile. Un discorso "estetico" o poco più - mentre quanto più a fondo si sarebbe dovuto scendere... [...] Non è estranea a tutto questo una mia grande timidezza di fronte a simili libri, oggi quasi incredibili e tanto più misteriosi quanto più sono puri... (Lettera di Cristina Campo a Gustaw Herling Grudziński, del 12 marzo 1961, AGHG 523, n. 1) ${ }^{11}$.

Mossa dalla meraviglia e dall'ammirazione, la scrittrice afferma di avere un unico scopo: quello di diffondere la conoscenza dei due racconti di Herling tra i lettori italiani, quelli veri: "Avevo anche molta fretta di far sapere alla gente che il libro c'era e che bisognava leggerlo. Del resto il vero lettore, il predestinato, non ha bisogno che di un cenno" (Lettera di Cristina Campo a Gustaw Herling Grudziński, del 12 marzo 1961, AGHG 523, n. 1). L'emozione suscitata in Cristina Campo dalla lettura dei racconti era così forte da farla perfino dichiarare a Herling che per più di un anno il suo libro era stato accanto solo a L'Aleph di Borges "e un altro di cui accenno nella nota" (si tratta del romanzo Il gesuita perfetto di Furio Monicelli), l'unico a darle il desiderio di scriverne.

Una settimana dopo Cristina Campo scrive una lettera a Leone Traverso, il noto grecista e germanista con cui la scrittrice aveva avuto una relazione nei primi anni Cinquanta. Anche in questa lettera Campo fa riferimento alla sua scoperta letteraria, con la speranza di destare lo stesso entusiasmo verso i racconti di Herling nel suo interlocutore:

Caro Leone,

ti ho fatto spedire da Gustaw Herling il suo strano e bellissimo libro Pale d'altare. Io ne ho fatta una nota sul "Punto", puramente orizzontale, però, intendo dire poco più che estetica - mentre il libro è di estrema verticalità. In certi momenti (vedrai lo stupendo racconto La torre) si apparenta un po' a Borges - con uno spirito però alla De Maistre, una cupa, integra disperazione di cattolico polacco.

Mi piacerebbe se tu lo segnalassi, magari brevemente, sul "Mattino" o all "“Approdo". Oltre a me, ne hanno parlato solo un paio di persone - come al solito per questi libri scandalosamente civili (Lettera di Cristina Campo a Leone Traverso [n. 100], del 22 marzo 1961, in: Campo, 2007, p. 111) ${ }^{12}$.

11 Alcune frasi di questo frammento sono riportate in Herling, 2019, p. CXLIII.

${ }_{12}$ In una delle lettere conservate presso l'Archivio di Gustaw Herling, Cristina Campo effettivamente chiede a Herling di mandare il libro a Leone Traverso. 
La richiesta della Campo ottenne immediatamente l'effetto sperato; e già il 20 aprile 1961 scrive a Herling riferendogli il contenuto della risposta di Traverso, in cui egli si dice "incantato dal suo libro [Pale d'altare]" (Lettera di Cristina Campo a Gustaw Herling, del 20 aprile 1961, AGHG 523, n. 4) $)^{13}$. Ne sarebbe nato un articolo di Leone Traverso su Herling, intitolato Il demone dell'analogia, pubblicato su "La Nazione" il 24 gennaio 1962.

Ma già prima della pubblicazione dell'articolo nel quotidiano fiorentino Traverso aveva mandato le bozze del suo testo a Cristina Campo, insieme a una cartolina con l'immagine di Urbino (dove risiedeva in quegli anni), in cui le esprimeva la sua gratitudine e ammirazione per Herling. Così Cristina Campo risponde a Traverso l'11 maggio 1961:

\begin{abstract}
Caro Leone,
grazie infinite - per Herling e per me. Il tuo articolo è di una ricchezza che solo al lettore più attento - più silenzioso - sarà dato di valutare. Ti mando, come desideri, la mia nota, ma solo perché tu veda quanto più lontano sei arrivato partendo da un modo di lettura quasi identico. In realtà il mio è un articolo del tutto orizzontale su cui bisognava, appunto, alzare una piramide.

Il "Mattino" o "L'Approdo" sono sedi ben ristrette per uno scritto come il tuo, ma se almeno sul giornale non hai limiti di spazio, vedi di includere 3-4 righe, accennando al motivo centrale dei racconti, se non proprio alla storia: il lebbroso, il sordomuto, il prete peccatore, i monaci murati nella Certosa ecc. Il che mi sembra importante per il tuo discorso.

Spedisco il tuo manoscritto a Herling che spero tu possa incontrare un giorno. È un uomo di un'estrema innocenza - non dolorosa (strano) ma piena di silenzio (Lettera di Cristina Campo a Leone Traverso [n. 101], del 11 maggio 1961, in: Campo, 2007, p. 112).
\end{abstract}

Come anticipato nella lettera, il dattiloscritto dell'articolo (Dattiloscritto della recensione di Pale d'altare di Gustaw Herling. AGHG 523, n. 9a, 901, 902, 903), insieme alla cartolina di Traverso (Cartolina

Giudicando dalle date, dovrebbe essere comunque una richiesta successiva, poiché la lettera è datata il 16 aprile 1961 (a meno che non si tratti di un errore nella datazione).

13 Il frammento riportatone nel Meridiano ("Traverso mi scrive che è incantato dal suo libro. Ne scriverà per l'Approdo e per il Mattino") è descritto con la data 30 giugno, vedi Herling, 2019, p. CXLII. 
di Leone Traverso nel carteggio della corrispondenza di Cristina Campo a Gustaw Herling, AGHG 523, n. 9), furono mandati dalla scrittrice a Herling e si trovano oggi nel suo archivio nel faldone delle lettere di Cristina Campo. Il dattiloscritto dell'articolo di Traverso comprende quattro fogli, con alcune correzioni inserite a penna con inchiostro blu: parole aggiunte o cambiate, un ampio frammento cancellato (nel terzo paragrafo riguardante il motivo di sogno), più altre minori correzioni. Sul retro del penultimo foglio del dattiloscritto è aggiunta una nota scritta a mano da Cristina Campo: "Con un affettuoso saluto da Cristina Campo", e probabilmente la stessa mano aggiunse l'indirizzo fiorentino di Traverso sotto il cognome di quest'ultimo, con cui il testo è firmato.

Come si può giudicare dalla versione finale e pubblicata dell'articolo, Traverso non seguì il consiglio della scrittrice per quanto riguarda l'eventuale inclusione dei personaggi e della storia, sottolineando invece l'allontanamento dello scrittore da un possibile procedimento narrativo verista che i grandi temi di entrambi racconti avrebbero potuto indurre. Traverso sottolinea inoltre il carattere universale delle storie raccontate: "alla fine quasi emblematicamente si ripropone al lettore il dramma dell'esistenza umana sulla terra, travolta da solitudine a colpa, da umiliazione a riscatto" (Traverso, 1962, p. 3). Il critico cita tre ampi frammenti dei racconti di Herling, tutti e tre focalizzati su questioni metafisiche ed esistenziali, come l'unicità o la ripetizione delle sorti umane ${ }^{14}$, la vita, la morte e il sogno, la colpa e la solitudine.

Per presentare Gustaw Herling nella prima parte del testo Traverso accenna ai tempi della guerra. Anche se nel 1958 esisteva già un'edizio-

14 Traverso inizia il suo articolo con un'ampia citazione da Pietà dell'isola: "Che la vita ripeta, come una ricamatrice privata del talento inventivo, modelli continuatamente simili tra loro, è cosa discussa fin da tempi immemorabili dai filosofi e non decisa fino ad oggi. Che le insondabili sentenze divine vengano scritte a migliaia di esemplari e modificate solo in varianti minime, o che, all'opposto, ciascuno di noi si rifletta nell'occhio immenso della Provvidenza e che dall'infallibile mano del Giudice riceva una sorte diversa e modellata esclusivamente a propria misura, è questione che preoccupa credenti e teologi” (Herling, 1962 citato in: Traverso, 1962, p. 3). Il tema delle matrici delle sorti umane che si ripetono risultava particolarmente caro allo scrittore polacco, vedi Herling, 2003, p. 135; Herling-Grudziński, 2018, p. 170. 
ne italiana di Un mondo a parte ${ }^{15}$, essa non era facilmente accessibile, e così per il critico, come per tanti altri lettori, la lettura di Pale d'altare rappresentava il primo contatto con l'opera di Herling. Traverso lo conferma, scrivendo:

L'autore, Gustav [sic] Herling, polacco, ha nell'ultima guerra mondiale sofferto in un campo di concentramento, e un libro tratto da quell'esperienza corre già tradotto in varie lingue. A noi bastano queste due novelle per intendere quanto sia familiare allo scrittore, con l'amore della vita, il senso inviolabile della solitudine e il pensiero della morte (Traverso, 1962, p. 3).

Per Traverso la cosa più importante per quanto riguarda la costruzione dei racconti è l'analogia. L'idea è congrua con la visione di Herling, per cui la citazione, la similitudine e l'analogia erano strumenti molto importanti per la costruzione della struttura narrativa (Jaworska, 2019, p. 1567) ${ }^{16}$. Alle analogie come mezzo tramite cui Herling realizza la tecnica della parabolizzazione accenna Arkadiusz Morawiec, individuando analogie intratestuali sul piano compositivo e analogie in forma di riferimenti intertestuali e intersemiotici sul piano intertestuale in senso lato (Morawiec, 2000, p. 170; Jaworska, 2019, p. 1569).

15 La prima edizione inglese è stata pubblicata nel 1951 con il titolo $A$ World Apart: $A$ Memoir of the Gulag. La prima edizione italiana è del 1958, ma la circolazione era alquanto limitata. Francesco Cataluccio racconta: "Il mio primo incontro con Herling, prima di diventargli amico, fu quando avevo quattordici anni e scovai casualmente, in una bancarella di libri usati vicino a Piazza San Marco a Firenze, una copia malmessa di Un mondo a parte (Inny świat), nella maltrattata edizione laterziana del 1958. Come ricordò amaramente Herling: «Laterza, editore di Croce stampò Un mondo a parte contro voglia; quasi per un obbligo, diciamo così, familiare. Dubito persino che l'abbia distribuito, visto che, girando per le librerie italiane, allora non ne vidi mai una copia. Ciò detto, con le sole eccezioni di Paolo Milano e di Leo Valiani, quel libro fu ignorato del tutto. E lo stesso accadde nel ' 65 quando, per volontà del compianto Domenico Porzio, il libro uscì da Rizzoli. Nessuna reazione: solo un bell'articolo di Giancarlo Vigorelli e una recensione su Paese Sera (di Gianni Toti) in cui si suggeriva alle autorità italiane di espellermi dall'Italia»" (Cataluccio, 2015).

16 Jaworska ricorre proprio a L'isola per illustrare l'uso dell'analogia da parte di Herling. 
Oltre che con Leone Traverso, Cristina Campo cercò di promuovere l'opera Herling anche con altri suoi interlocutori. In una lettera precedente, datata 14 febbraio 1961, la Campo aveva chiesto a Herling di mandare il libro a Margherita Guidacci: "Spero possa recensirlo sul «Popolo». Dovrebbe piacerle molto (forse lei conosce le sue poesie)" (Lettera di Cristina Campo a Gustaw Herling, del 14 febbraio 1961, AGHG 523, n. 2) ${ }^{17}$. La speranza si realizza e il 26 maggio 1961 esce sul "Popolo" l'articolo di Margherita Guidacci, intitolato Lo scrittore polacco, in cui la poetessa fa un resoconto della lettura dei due racconti di Herling, individuando come motivo più importante il pellegrino di pietra della montagna di Santa Croce. I personaggi descritti ne La torre e nella Pietà dell' isola diventano così, secondo Guidacci, successive incarnazioni della figura del pellegrino di roccia. Margherita Guidacci riassume poi abbastanza dettagliatamente e in chiave narrativa le sorti dei protagonisti della Torre (lasciando da parte il secondo racconto): Pier Bernardo Guasco e il vecchio professore venuto da Messina. Alla fine si fa riferimento alla questione della religiosità di Herling come scrittore che "sarebbe improprio chiamare religioso - perché ci lascia necessariamente perplessi una religiosità in cui ha tanta parte, peggio ancora che la disperazione, la non speranza, che è il clima in cui respirano il Pellegrino e i suoi fratelli delle varie epoche" (Guidacci, 1961, p. 5).

Nella stessa lettera la Campo accenna alla recensione di Elémire Zolla (Lettera di Cristina Campo a Gustaw Herling del 14 febbraio 1961, AGHG 523, n. 2) che, così come il futuro commento di Leone Traverso, lei giudica "verticale", contrariamente alla propria recensione, descritta da lei come "orizzontale". Il testo di Zolla fu pubblicato sulla "Gazzetta del Popolo" proprio il 14 marzo $1961^{18}$. Nel suo articolo La catena dei mali, Zolla fa riferimento a Un mondo a parte, definendolo il libro sui campi russi più patetico e tremendo fra quanti si siano potuti leggere. La raccolta Pale di altare è definita una meditazione sul destino

17 In una delle lettere successive Cristina Campo scrive invece: "La mia copia l'ho data a Margherita Guidacci”, Lettera di Cristina Campo a Gustaw Herling del 16 aprile 1961, AGHG 523, n. 3.

18 E. Zolla, La catena dei mali, "Gazzetta del Popolo” del 14 marzo 1961. 
e sul martirio. Particolarmente interessante agli occhi di Zolla risulta il personaggio di Padre Rocca del racconto Pietà dell'isola, il quale, attraverso il proprio sacrificio, riesce a rompere l'eponima catena dei mali. Zolla sostiene che nel libro il motivo del sogno abbia un particolare rilievo. Afferma anche che Herling, come Hoffmannstahl, riesce a scoprire il mistero nell'ovvio e a contemplare il destino. Il destino in questa prospettiva è visto come il garbuglio delle necessità nella vita, il labirinto in cui i tre fili annodati - la morte, la risurrezione e il sacrificio - diventano il filo d'Arianna. Herling-Grudziński fu molto contento dell'articolo di Zolla, come si vede dalla lettera inviata il 17 marzo 1961 a Jerzy Giedroyć (Herling-Grudziński, 2019, pp. 290-291). Nella lettera Zolla è definito "indubbiamente uno dei più intelligenti critici letterari della giovane generazione nell'Italia di oggi" (Herling-Grudziński, 2019 , p. 291). Con la lettera lo scrittore manda a Giedroyć la recensione di Zolla insieme a quella della Campo, dicendo che tutte e due sono perfette "ma più accattivante e più penetrante è quella su «Il Punto»" (Herling-Grudziński, 2019, p. 290), cioè la recensione firmata da Cristina Campo.

Nello stesso periodo anche Roberto Calasso dedica un testo a Herling. In questo caso è possibile che lo stimolo per scrivere su Herling sia partito da Elémire Zolla ${ }^{19}$ che all'inizio degli anni 60 conobbe Calasso come suo studente e ne rimase impressionato. "Il giovane Calasso è bersagliato dai redattori - anche a Tempo Presente volevano modificargli, accorciargli, «adattargli» l'articolo. Ha rifiutato - speriamo a proprio vantaggio", scrive Cristina Campo a Gustaw Herling il 16 aprile 1961. Per poi aggiungere qualche giorno dopo: "Calasso fa benissimo a non toccare il suo articolo - quando lo vedrà capirà perché” (Lettera di Cristina Campo a Gustaw Herling, del 20 aprile, AGHG 523, n. 4). La recensione del volume Pale d'altare scritta da Roberto Calasso e intitolata Lebbra e solitudine uscì su "Tempo Presente" nel 1961, n. 4-5 (aprile-maggio). Calasso individua subito l'inclinazione me-

19 Come viene fuori da una delle lettere di Zolla a Herling. Attualmente sto preparando un contributo sulla corrispondenza inedita di Elémire Zolla e Gustaw Herling, in cui intendo approfondire il loro rapporto. 
tafisica come motivo chiave del volume, che "è il tentativo di tenere gli occhi fissi in un contatto inesorabile su questi «mali metafisici»" (Calasso, 1961, p. 370). Poi si concentra su questioni più universali ("L'universo di Herling è l'universo mitologico della colpa" [Calasso, 1961, p. 371]), individuando nei racconti lo stesso substrato esistenziale: "si narra di chi ha passato la propria vita a prepararsi alla prova suprema e, quando questa si presenta, cede e si ritrae" (Calasso, 1961, p. 370). Riferendosi a strutture generali, Calasso rinuncia alla necessità di individuare un qualche motivo principale e a ricapitolare le sorti del personaggio, per dichiarare invece: "Riassumere Herling è inutile" (Calasso, 1961, p. 370). Dal momento che, secondo l'interpretazione di Calasso, i racconti di Pale d'altare, sfociano in una meditazione liturgica, "il movimento della trama è solo un velo che cela la fissità atroce del fondo" (Calasso, 1961, p. 370).

I contatti cordiali tra Herling e il giovane Calasso sono testimoniati dal carteggio con Cristina Campo, dove si può trovare anche una cartolina del 1964, firmata da lei, Elémire Zolla, Roberto Calasso e Mario Bortolotto (Cartolina di Cristina Campo a Gustaw Herling, AGHG 523, n. 6). Gli amici romani di Gustaw Herling gli mandano un affettuoso ricordo, dicendo che rimangono in attesa del Secondo Avvento. La prima traduzione italiana del racconto di Herling Il secondo avvento sarebbe stata pubblicata nel 1965 sulla rivista "Elsinore" (Herling, 1965).

L'amicizia di Cristina Campo e Gustaw Herling è testimoniata dalla loro corrispondenza anche negli anni successivi. Nel 1964 (Cartolina di Cristina Campo a Gustaw Herling del 14 febbraio $1964^{20}$, AGHG 523, n. 7) Cristina Campo manda a Herling un invito da parte di Vanni Scheiwiller (Lettera di Vanni Scheiwiller, AGHG 523, n. 7a) a curare un volume di poesie di Anna Achmatova, sul modello di quello di William Carlos Williams curato dalla stessa Cristina Campo (Williams, 1958).

Gustaw Herling fa riferimento alla scrittrice italiana in un suo saggio incentrato sul famoso scritto di Simone Weil Venezia salva, tradotto

20 La data viene confermata anche dal timbro postale sulla busta che conteneva la cartolina di Cristina Campo e la lettera di Vanni Scheiwiller (AGHG 523, n. 7b). 
da Cristina Campo ${ }^{21}$. Nel 1977, dopo la morte precoce della scrittrice, Herling scrive: "Incompiuta eppure bellissima, tradotta in un modo esemplare in italiano dalla compianta Cristina Campo (e pubblicata nel

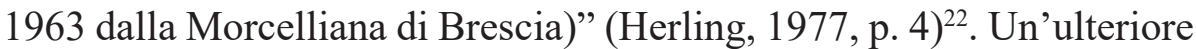
testimonianza dell'affinità spirituale tra i due scrittori da affiancare idealmente al loro carteggio, insieme ai testi delle recensioni ai racconti di Herling composte grazie all'entusiasmo di Cristina Campo verso la sua opera.

Le lettere, finora inedite, di Cristina Campo a Gustaw HerlingGrudziński, oltre a documentare la loro amicizia, offrono una testimonianza esemplare dell'entusiasmo disinteressato della grande scrittrice. Insieme al fondamentale testo critico della Campo su Pale d'altare e alle recensioni legate al sodalizio con Herling queste lettere rappresentano un momento importante e, nello stesso tempo, troppo poco conosciuto, delle relazioni letterarie italo-polacche.

\section{BIBLIOGRAFIA}

Calasso, R. (1961). Lebbra e solitudine. Tempo Presente, 4-5 (aprile-maggio), $370-371$.

Campo, C. (1971). Il flauto e il tappeto. Milano: Rusconi.

Campo, C. (1987). Gli imperdonabili. Milano: Adelphi.

Campo, C. (1990). La torre e l'isola. In G. Herling, Due racconti. La torre.

Il miracolo, con uno scritto di Cristina Campo e nove disegni di Jan Lebenstein (pp. 9-17). Milano: Libri Scheiwiller.

Campo, C. (1991). La Tigre Assenza. Milano: Adelphi.

Campo, C. (1992). La tour et l'île (translated by J.-B. Para). In G. Herling, L'île: et autres récits (translated by T. Douchy) (pp. 11-17). Paris: Gallimard.

Campo, C. (1998). La torre e l'isola. In C. Campo, Sotto falso nome, a cura di M. Farnetti. Milano: Adelphi.

21 Simone Weil apparteneva alle più grandi fascinazioni letterarie e mistiche di Cristina Campo. Vedi tra l'altro Negri, 2005 e la bibliografia indicata nel volume.

22 Cristina Campo è morta lo stesso anno, 1977, il 10 gennaio. Come traduttrice di Venezia salva di Simone Weil è stata nominata anche nel 1964 su "Kultura" parigina, dove Herling ha pubblicato il saggio Wenecja ocalona in polacco, vedi Herling, 1964. 
Campo, C. (1998). Lettere a un amico lontano. Milano: Libri Scheiwiller. Campo, C. (1998). Sotto falso nome, a cura di M. Farnetti. Milano: Adelphi.

Campo, C. (1999). Lettere a Mita, a cura e con nota di M. Pieracci Harwell. Milano: Adelphi.

Campo, C. (2007). Caro Bul. Lettere a Leone Traverso (1953-1967), a cura e con nota di M. Pieracci Harwell. Milano: Adelphi.

Campo, C. (2009). Se tu fossi qui. Lettere a Maria Zambrano 1961-1975, a cura di M. Pertile. Milano: Archinto.

Campo, C. (2010). Un ramo già fiorito. Lettere a Remo Fasani, a cura di M. Pertile. Venezia: Marsilio.

Campo, C. (2011). Il mio pensiero non vi lascia. Lettere a Gianfranco Draghi e ad altri amici del periodo fiorentino. Milano: Adelphi.

Campo, C., \& Spina, A. (2007). Carteggio. Brescia: Morcelliana.

Cataluccio, F. (2015, Feb. 25). Uno scrittore a parte. Il Post. Retrieved from www.ilpost.it/francescocataluccio/2015/02/25/gustaw-herling.

Craveri, P. (2015) Riflessioni e testimonianze. In M. Herling, L. Marinelli (Eds.), Dall' 'Europa illegale' all'Europa unita. Gustaw HerlingGrudziński: L'uomo, lo scrittore, l'opera. Roma: Accademia Polacca delle Scienze.

De Stefano, C. (2002). Belinda e il mostro. Vita segreta di Cristina Campo. Milano: Adelphi.

Dickinson, E. (1953). Quattro poesie: Tocca leggero la dolce chitarra; Che farò io quando turba l'estate; Che tedio attendere; Morte è il pieghevole corteggiatore (translated by V. Guerrini). Corriere dell'Adda, 2 maggio.

Farnetti, M. (1996). Cristina Campo. Ferrara: Luciana Tufani.

Guidacci, M. (1961). Lo scrittore polacco. Il Popolo, 26 maggio, 5.

Herling, G. (1960). Pale d'altare, a cura di D. Staffa e P.F. Poli. Parma: Silva. Herling, G. (1964). Wenecja ocalona. Kultura, 3 (197), 25-37.

Herling, G. (1965). Il secondo avvento (translated by M. Lenzi). Elsinore, $14-5,5-25$.

Herling, G. (1977). "Venezia salva”, cara a Silone. Il Giornale Nuovo, Milano, 17 marzo, 4.

Herling, G. (2003). La mia "isola". Conversazione con Włodzimierz Bolecki, a cura di M. Herling. In G. Herling, L'isola (translated by D. Tozzetti). Napoli: l'ancora del mediterraneo.

Herling, G. (2019). Etica e letteratura. Testimonianze, diario, racconti, a cura di K. Jaworska. Milano: Mondadori. 
Herling, M. (2019). Cronologia. In G. Herling. Etica e letteratura. Testimonianze, diario, racconti, a cura di K. Jaworska (pp. XCIII-CLXII). Milano: Mondadori.

Herling-Grudziński, G. (1992). Diario scritto di notte, trad. it. di D. Tozzetti, scelta dei brani a cura di G. Herling e F. Cataluccio. Milano: Feltrinelli. Herling-Grudziński, G. (2012). Dziennik pisany noca, vol. 3 (1993-2000). Kraków: Wydawnictwo Literackie.

Herling-Grudziński, G. (2018). Rozmowy w Dragonei. Rozmowy w Neapolu. In G. Herling-Grudziński, Dzieła zebrane, vol. 11, edited by W. Bolecki. Kraków: Wydawnictwo Literackie.

Herling-Grudziński, G. (2019). Jerzy Giedroyć. Korespondencja, vol. 1. 1944 -1966. In G. Herling-Grudziński, Dzieła zebrane, vol. 12, edited by W. Bolecki. Kraków: Wydawnictwo Literackie.

Jaworska, K. (2019). Apparati: Diario scritto di notte. In Herling, G. Etica e letteratura. Testimonianze, diario, racconti, a cura di K. Jaworska (pp. 1511-1642). Milano: Mondadori.

Morawiec, A. (2000). Poetyka opowiadań Gustawa Herlinga-Grudzińskiego. Kraków: Universitas.

Mughini, G. (2019). Sia lode a Herling. Il Foglio, 29 ottobre. Retrieved from https://www.ilfoglio.it/uffa/2019/10/29/news/sia-lode-a-herling-283463/

Negri, F. (2005). La passione della purezza. Simone Weil e Cristina Campo. Padova: Il Poligrafo.

Pertignani, S. (2012). Addio a Roma. Venezia: Neri Pozza.

Saviano, R. (2009). Mai più in un mondo a parte. In R. Saviano. La bellezza e l'inferno. Scritti 2004-2009, pp. 223-231. Milano: Mondadori.

Śniedziewska, M. (2019). “Osobiste sprawy i tematy”. Gustaw Herling wobec dwudziestowiecznej literatury włoskiej. Warszawa: IBL PAN.

Traverso, L. (1962). Il demone dell'analogia. La Nazione, 24 gennaio, 3.

Weil, S. (1963). Venezia salva, trad. it. e cura di C. Campo. Brescia: Morcelliana.

Weil, S. (1974 [1967]). L'Iliade o il poema della forza (translated by C. Campo). In S. Weil, La Grecia e le intuizioni precristiane, a cura di C. Campo e M. Pieracci Harwell (pp. 9-44). Milano: Rusconi.

Williams, W.C. (1958). Il fiore è il nostro segno. Poesie (translated by C. Campo). Milano: All'Insegna del Pesce d'Oro.

Zolla, E. (1961). La catena dei mali. Gazzetta del Popolo, 14 marzo. 


\section{Lettere del carteggio di Cristina Campo a Gustaw Herling-Grudziński}

Le lettere inedite di Cristina Campo a Herling fanno parte dell'Archivio di Gustaw Herling, custodito presso la Fondazione "Biblioteca Benedetto Croce". Le lettere hanno la collocazione AGHG 523 (Katalog Archiwum Gustawa Herlinga-Grudzińskiego, a cura di J. Borysiak, Biblioteka Narodowa, Warszawa 2019).

Desidero ringraziare Marta Herling, responsabile dell'Archivio, e gli eredi degli scritti di Cristina Campo, Maria Pertile, Luciana Bragaglia, Roberto Calasso, per avermi gentilmente concesso l'autorizzazione a pubblicare le citazioni riportate nell'articolo.

1) Lettera di Cristina Campo a Gustaw Herling-Grudziński del 12 marzo 1961, AGHG 523, n. 1. Manoscritto a penna blu su carta intestata CRISTINA CAMPO. La busta che conteneva la lettera, AGHG 523, n. 1a. Sulla busta timbro postale: Roma Ferrovia 15-III-1961. Sul retro della busta timbro con la data di arrivo: Napoli 16-III-1961.

2) Lettera di Cristina Campo a Gustaw Herling-Grudziński del 14 febbraio 1961, AGHG 523, n. 2.

3) Lettera di Cristina Campo a Gustaw Herling-Grudziński del 16 aprile 1961, AGHG 523, n. 3. Manoscritto a penna blu su carta intestata: Cristina Campo.

4) Lettera di Cristina Campo a Gustaw Herling-Grudziński del 20 aprile 1961, AGHG 523, n. 4.

5) Cartolina a Gustaw Herling-Grudziński del 3 dicembre 1961, AGHG 523, n. 6. Cartolina: un dettaglio del bassorilievo di Luca della Robbia, Firenze, Museo S. Maria del Fiore. Sulla cartolina timbro postale: Roma Ferrovia 3-XII-1961.

6) Cartolina di Cristina Campo a Gustaw Herling-Grudziński del 14 febbraio 1964, AGHG 523, n. 7. Cartolina: Roma, Castel Sant'Angelo.

7) Lettera di Vanni Scheiwiller a Cristina Campo allegata alla Cartolina di Cristina Campo a Gustaw Herling del 14 febbraio 1964 (n. 7), AGHG 523, n. 7a. La busta che conteneva la lettera n. 7a e la cartolina n. 7, AGHG 523, n. 7 b. Sulla busta timbro postale: 14-II-1964.

7) Cartolina di Cristina Campo a Gustaw Herling-Grudziński del 28 ottobre 1964, AGHG 523, n. 8. Cartolina: l'immagine del dettaglio della Vida de San Vincent, Museo de Cataluña (Barcelona). Sulla cartolina timbro postale: Poste Roma Ferr., 28.10.64. 


\section{8) Cartolina di Leone Traverso a Cristina Campo nel carteggio della corrispon- denza di Cristina Campo a Gustaw Herling, AGHG 523, n. 9. Cartolina: Urbino, Palazzo Ducale, I Torricini, sec. XV. \\ 9) Dattiloscritto della recensione di Leone Traverso di Pale d'altare di Gustaw Herling. AGHG 523, n. 9a, 901, 902, 903.}

Riassunto: Nell'Archivio di Gustaw Herling-Grudziński a Napoli sono conservate le lettere di Cristina Campo allo scrittore che sono una testimonianza di una relazione letteraria nata tra loro intorno alla raccolta di racconti di Gustaw Herling-Grudziński Pale d'altare, tradotta in italiano e pubblicata in Italia nel 1960. Cristina Campo, poetessa, saggista e traduttrice, una figura di grande rilievo del Novecento letterario italiano, anche se ancora sconosciuta in ambito polacco, ha dedicato all'opera di Herling-Grudziński una recensione intitolata La torre e l'isola, pubblicata su "Il Punto" nel 1961, fondamentale per la fortuna dell'opera di Herling-Grudziński in Italia e che lo scrittore stesso ha considerato uno dei testi critici più importanti a lui dedicati. L'articolo si pone come obiettivo di rendere conto del ruolo e dell'importanza di Cristina Campo nella ricezione dell'opera di Gustaw Herling-Grudziński in Italia, nonché di presentare il rapporto che tra lo scrittore polacco e la sua critica italiana attraverso le lettere inedite della Campo a Herling-Grudziński. Il contributo fa riferimento anche a altri testi critici su Pale d'altare, che furono scritti su stimolo della Campo, come quelli di Elèmire Zolla, Roberto Calasso, Margherita Guidacci e Leone Traverso.

Parole chiave: Cristina Campo, Gustaw Herling-Grudziński, Pale d'altare, corrispondenza, ricezione di letteratura straniera in Italia 